\title{
Phosphorylation of histone H3 correlates with transcriptionally active loci
}

\author{
Scott J. Nowak and Victor G. Corces ${ }^{1}$ \\ Department of Biology, The Johns Hopkins University, Baltimore, Maryland 21218, USA
}

\begin{abstract}
Posttranslational modifications of the $\mathrm{N}$-terminal tails of the core histones within the nucleosome particle are thought to act as signals from the chromatin to the cell for various processes. The experiments presented here show that the acetylation of histones $\mathrm{H} 3$ and $\mathrm{H} 4$ in polytene chromosomes does not change during heat shock. In contrast, the global level of phosphorylated H3 decreased dramatically during a heat shock, with an observed increase in $\mathrm{H} 3$ phosphorylation at the heat shock loci. Additional experiments confirm that this change in phosphorylated $\mathrm{H} 3$ distribution is dependent on functional heat shock transcription factor activity. These experiments suggest that $\mathrm{H} 3$ phosphorylation has an important role in the induction of transcription during the heat shock response.
\end{abstract}

[Key Words: Histone phosphorylation; histone acetylation; transcription; heat shock, chromatin]

Received September 6, 2000; revised version accepted October 16, 2000.

The eukaryotic genome is highly compacted within the nucleus into the chromatin fiber. The basic organizational unit of the chromatin fiber, the nucleosome, consists of the DNA molecule wound around an octamer comprised of the core histones $\mathrm{H} 2 \mathrm{~A}, \mathrm{H} 2 \mathrm{~B}, \mathrm{H} 3$, and $\mathrm{H} 4$ (Wolffe 1998). The positively charged, unstructured $\mathrm{N}$ terminal portions of these core histones protrude from the nucleosome core where they are subject to covalent modification by a variety of cellular factors. These covalent modifications of the $\mathrm{N}$-terminal tail domains are proposed to act as signals from the DNA to the cellular machinery for various processes including transcription, chromosomal condensation, and mitotic segregation (Strahl and Allis 2000).

The acetylation of the $\mathrm{N}$-terminal tails is the beststudied modification of the core histones. Several transcription factors, such as GCN5 (Trievel et al. 1999), and the $\mathrm{TAF}_{\mathrm{II}} 250$ subunit of TFIID (Mizzen et al. 1996), as well as subunits of the RNA polymerase complex (Wittschieben et al. 1999) have intrinsic histone acetyltransferase (HAT) activity, which suggests a potential role for histone acetylation in either the activation or maintenance of transcription. The acetylation of the $\mathrm{N}$ terminal tail domains of core histones $\mathrm{H} 3$ and $\mathrm{H} 4$ at various lysine residues is essential for the normal implementation of various cellular processes, such as promoter-transcription factor association (Vettese-Dadey et al. 1996; Jacobson et al. 2000), gene transcription (Mizzen and Allis 1998), and dosage compensation (Turner 1991; Smith et al. 2000).

${ }^{1}$ Corresponding author.

E-MAIL corces@jhu.edu; FAX (410) 516-5456.

Article and publication are at www.genesdev.org/cgi/doi/10.1101/ $\operatorname{gad} .848800$.
Phosphorylation of serine 10 of the $\mathrm{N}$-terminal arm of histone $\mathrm{H} 3$ has been shown to be essential for proper mitotic chromosomal condensation (Hendzel et al. 1997; Wei et al. 1998) and segregation (Wei et al. 1999). In addition, recent studies have outlined the possibility that histone $\mathrm{H} 3$ phosphorylation may have a role in the regulation of transcription. Ser $10 \mathrm{H} 3$ phosphorylation is found to rapidly increase in quiescent cells during mitogenic stimulation (Barratt et al. 1994), as well as during immediate-early gene induction via the epidermal growth factor (EGF)-signaling pathway (Mahadevan et al. 1991). In addition, recent experiments performed in vitro have suggested that EGF-stimulated $\mathrm{H} 3$ phosphorylation may act as a signal for histone acetyltransferase binding and subsequent acetylation of a particular locus during transcription initiation (Cheung et al. 2000; Lo et al. 2000).

The heat shock response in the fruit fly (Drosophila melanogaster) is a well-characterized model for the induction of gene expression in response to environmental stress (Ashburner and Bonner 1979; Pauli et al. 1992). Briefly, during a heat shock, transcription (McKenzie et al. 1975; Spradling et al. 1975) and translation (Mirault et al. 1978) of most normally expressed cellular gene products in Drosophila cells cease. This cessation of normal gene expression is accompanied by the rapid induction and expression of the heat shock genes (McKenzie et al. 1975; Spradling et al. 1975; Mirault et al. 1978).

Examination of the puffing pattern of polytene chromosomes isolated from larval salivary glands indicates that this repression of normal gene expression during a heat shock is accompanied by a reduction in size of the normal developmental and ecdysone-induced puffs (Ashburner 1970, 1972), sites that are known to contain ac- 
tively transcribing loci (Spradling et al. 1975). Induction of the heat shock genes is accompanied by a characteristic and reproducible puffing of the chromosomal subdivisions that contain the heat shock genes (Ashburner 1970). By examining polytene chromosomes using immunocytochemical methods, the heat shock response therefore allows us to examine if any changes might occur in the distribution of acetylated or phosphorylated core histones within the genome during a controlled alteration of transcription.

Here we report that the number and distribution of genes containing Ser 10 phosphorylated histone H3 change drastically in response to heat shock and that the change in distribution is dependent on the presence of a functional heat shock transcription factor. We further determine that heat shock has no detectable effect on the presence or distribution of acetylated $\mathrm{H} 3$ and $\mathrm{H} 4$, which suggests that change in this modification may not be required for the activation or repression of transcription that accompanies the heat shock response.

\section{Results}

Acetylation of histones $\mathrm{H} 3$ and $\mathrm{H} 4$ does not change substantially in response to heat shock

Acetylation of core histones H3 and H4 at lysines 14 and 8 , respectively, has been linked to gene transcription (Kuo et al. 1996; Nightingale et al. 1998; Berger 1999; Cheung et al. 2000; Strahl and Allis 2000). In addition, deacetylation of core histones is thought to have a role in silencing specific loci (Nan et al. 1998). We were therefore particularly interested in determining whether the heat shock response of $D$. melanogaster would produce a change in the distribution of acetylated histones $\mathrm{H} 3$ and $\mathrm{H} 4$. Because of the near-total repression of cellular gene products during a heat shock, we might expect that the distribution of acetylated $\mathrm{H} 3$ and $\mathrm{H} 4$ would radically change during thermal stress in a manner reflective of the transcriptional profile of the cell (Spradling et al. 1975; Mirault et al. 1978; Lindquist 1986). Because acetylation of $\mathrm{H} 3$ at Lys 14 of the $\mathrm{N}$-terminal arm has been described as essential for transcription /Cheung et al. 2000; Clayton et al. 2000), we first examined the distribution of acetylated H3 by staining polytene chromosomes with an antibody specific for Lys 14 acetylated histone H3 (Boggs et al. 1996). Lys 14 acetylated H3 staining was observed at the puffs, which are active sites of transcription in polytene chromosomes (Spradling et al. 1975; Bonner and Pardue 1977), and distributed throughout the chromosomes in discrete bands before heat shock (Fig. 1A,C,E). One locus that we examined, subdivision $62 \mathrm{~A}$, which becomes puffed during larval development in response to ecdysone (Ashburner 1972), is intensely labeled with the Lys 14 acetylated H3 antibody (Fig.1A,E). In addition, other chromosomal subdivisions such as 89B display Lys 14 acetylated H3 staining but are not puffed before heat shock (Fig. 1A,E). The chromosomal subdivision 93D, which is known to become puffed during heat shock, was Lys 14 acetylated but not puffed before heat shock (Fig. 1A,E). Examination of polytene chromosomes from larvae that were subjected to a 20-min heat shock showed that the 87A and $87 \mathrm{C}$ heat shock puffs, which contain the hsp 70 gene cluster (Livak et al. 1978), were stained by the anti-Lys 14 acetylated antibody, although the staining at these puffs appeared to be less intense and rather diffuse (Fig. 1B,D,F). This might not represent a reduction in the level of acetylation, but rather a decrease in signal intensity due to the large puffing at the heat shock loci. After heat shock, the overall number of discrete stained bands did not appear to change significantly and regions that were stained before heat shock, such as $89 \mathrm{~B}$, remained acetylated (Fig. 1B,F). Loci with acetylated $\mathrm{H} 3$ staining that were puffed before heat shock, such as $62 \mathrm{~A}$, were no longer puffed after heat shock but remained acetylated (Fig. 1B,F). The observation that the heat shock genes are acetylated before heat shock, at a time when they are not transcribed, and non-heat shock genes, which are not transcribed during heat shock, are acetylated during heat shock, suggests that the presence of Lys 14-acetylated H3 does not necessarily denote an actively transcribed locus.

Recent examination of $\mathrm{H} 3$ acetylation during EGF stimulation raises the issue that antibodies against Lys 14 acetylated $\mathrm{H} 3$ may show decreased recognition of their epitope when other modifications, such as phosphorylation, coexist on the same histone tail (Clayton et al. 2000). This problem can be overcome by using antibodies against histone $\mathrm{H} 3$ acetylated at lysines 9 and 14 . To ensure that our results were not caused by this potential artifact, we examined the distribution of hyperacetylated $\mathrm{H} 3$ using antibodies against $\mathrm{H} 3$ acetylated at lysines 9 and 14 on the N-terminal tail (Hendzel et al. 1997) before (Fig. 2A) and after (Fig. 2B) heat shock. The results suggest that the distribution of diacetylated $\mathrm{H} 3$ is similar to the distribution of Lys 14 acetylated H3 before and after heat shock. Diacetylated H3 staining appears to be more widespread than monoacetylated staining, which is probably caused by the antibody's recognition of acetylation of $\mathrm{H} 3$ at lysine 9. The intensity of staining of the Lys 9,14-acetylated H3 antibody at several of the heat shock puffs examined appears to be similar to that observed with the Lys 14-acetylated H3 antibody (Fig. 2B). These results suggest that the diffuse staining at the heat shock puffs is not an artifact attributed to the masking of the acetylated Lys 14 epitope by Ser 10 phosphorylation.

$\mathrm{H} 4$ acetylation was also examined using antibodies specific for Lys 8-acetylated histone H4 (Boggs et al. 1996; Vettese-Dadey et al. 1996) to stain polytene chromosomes isolated from third instar larvae. The distribution of Lys 8 acetylated histone $\mathrm{H} 4$ was similar to that of acetylated $\mathrm{H} 3$, with $\mathrm{H} 4$ acetylation observed in discrete bands in nonpuffed regions, such as subdivision 89B, and at ecdysone-induced puffed regions, such as $62 \mathrm{~A}$, before heat shock (Fig. 2C). Chromosomal subdivisions 87A and $87 \mathrm{C}$, which contain the hsp 70 heat shock genes (Livak et al. 1978), were acetylated before (Fig. 2C) and after heat shock (Fig. 2D). Similar to acetylated H3, heat 

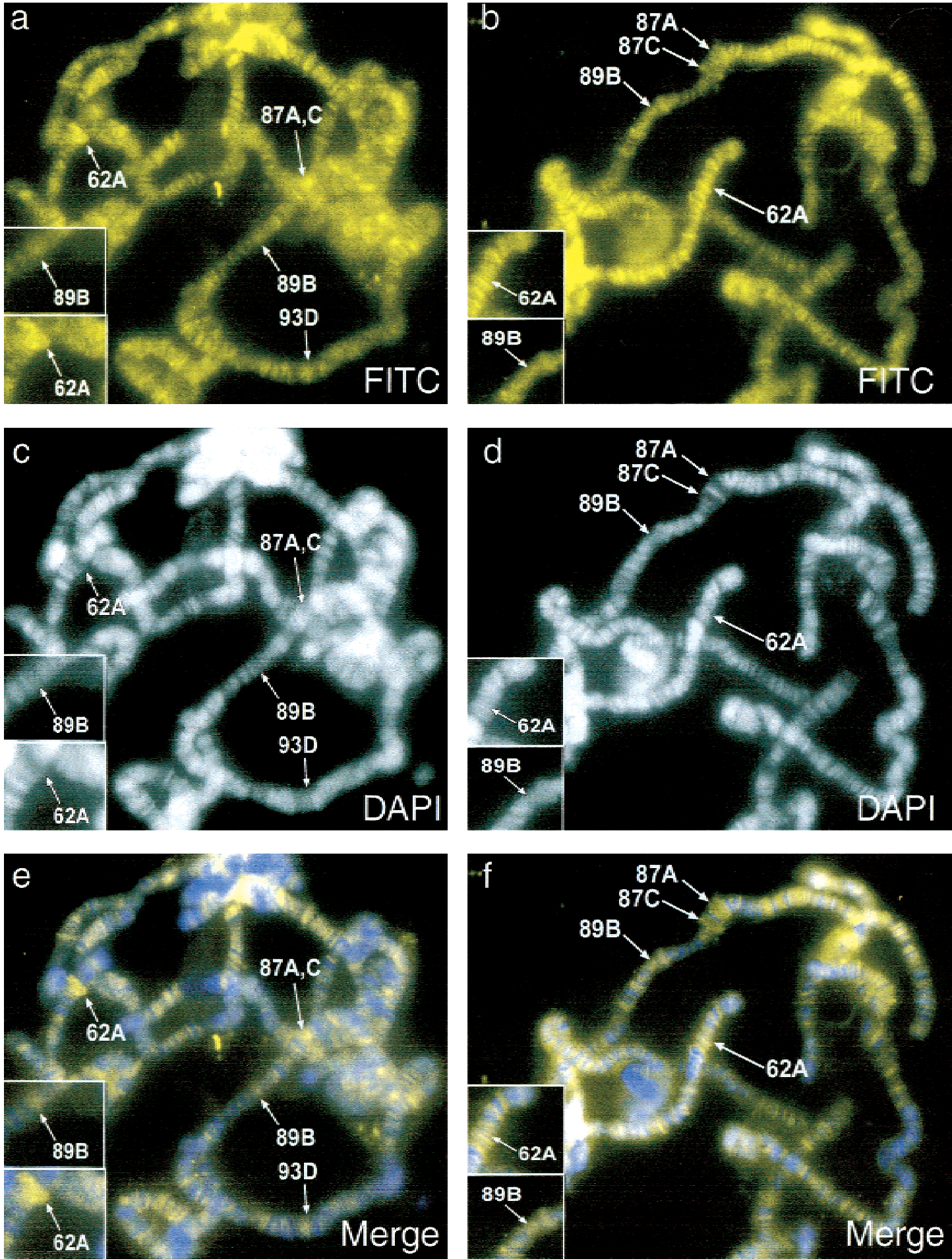

Figure 1. Distribution of Lys 14 acetylated histone $\mathrm{H} 3$ does not change during heat shock. $(A, B)$ FITC image of anti-Lys 14 acetylated H3 immunostaining. $(C, D)$ 4',6-diamidino-2-phenylindole (DAPI) counterstaining of chromosomes shown in $A$ and $B$. (E, $F$ ) Composite merge of FITC and DAPI channels, antibody staining shown in yellow, DAPI shown in blue. $(A, C, E)$ Antibodies specific for Lys 14 acetylated $\mathrm{H} 3$ stain multiple discrete sites in polytene chromosomes prepared from third instar larvae grown at $22^{\circ} \mathrm{C}$. $(C, E)$ Ecdysoneinduced developmental puff 62A and nonpuffed chromosomal subdivision 89B both display Lys 14 acetylated $\mathrm{H} 3 \mathrm{staining}$ (insets). Heat shock locus 93D is acetylated but not puffed. $(B, D, F)$ Anti-Lys 14 acetylated $\mathrm{H} 3$ staining after heat shock is observed at the $87 \mathrm{~A}$ and $87 \mathrm{C}$ puffs, which contain the hsp70 gene cluster. $(B, F)$ Chromosomal subdivisions 89B and 62A, which do not contain heat shock genes, are not puffed after heat shock but contain histones that remain acetylated (insets). 

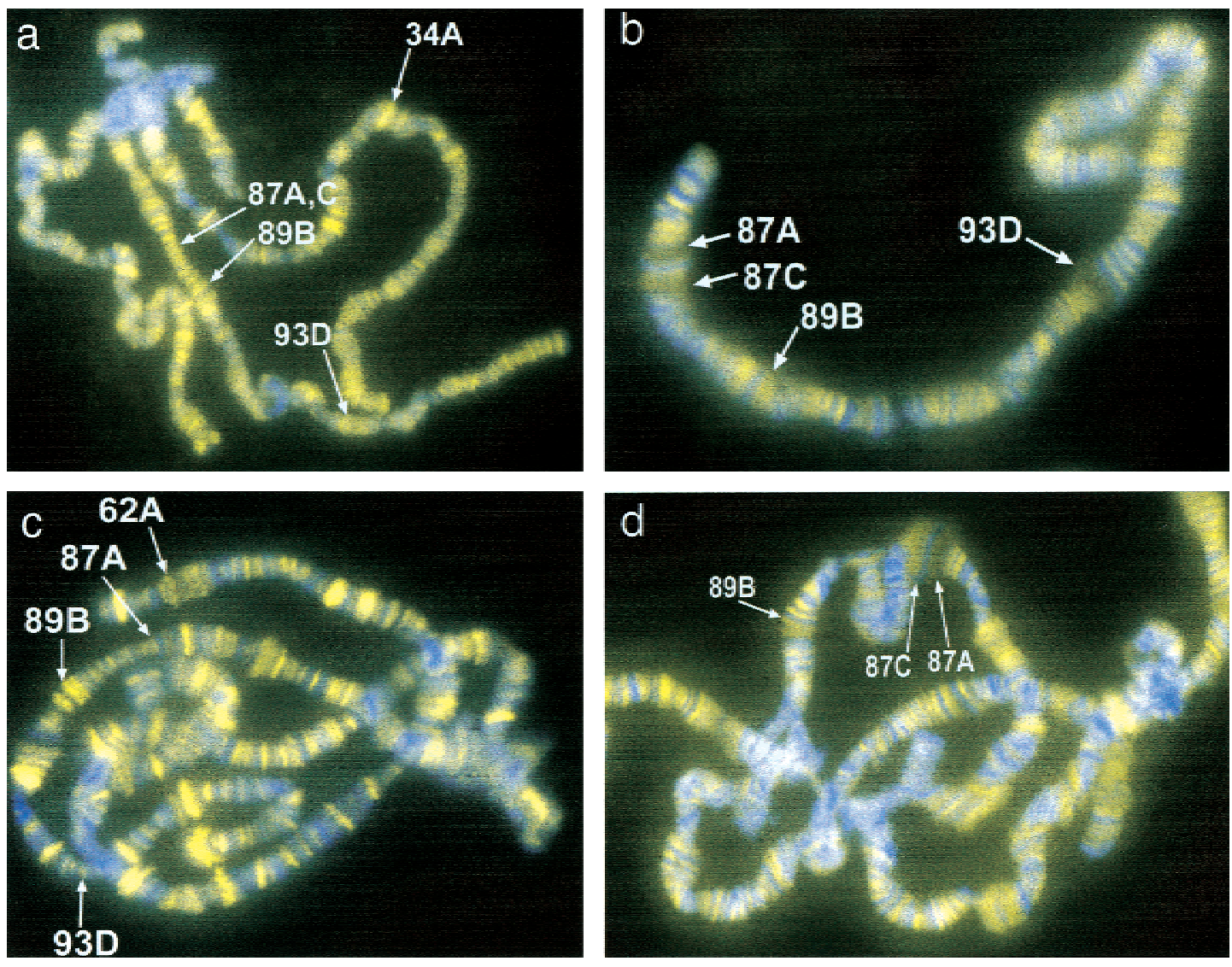

Figure 2. Distribution of diacetylated histone $\mathrm{H} 3$ and Lys 8 acetylated $\mathrm{H} 4$ does not change during heat shock. In all panels, antibody staining is shown in yellow, chromosomes are counterstained with 4',6-diamidino-2-phenylindole (DAPI; blue). (A) Antibodies specific for $\mathrm{H} 3$ acetylated at lysines 9 and 14 stain region 89B, the developmental puff at 34A, and loci 87A, 87C, and 93D, which contain heat shock genes before heat shock. (B) Fragment of chromosome 3R isolated from heat shocked third instar larva showing the 87A, 87C, and 93D heat shock puffs, stained with anti-Lys 9,14 acetylated H3. Subdivision 89B, which does not contain any heat shock genes, is acetylated but not puffed after heat shock. (C) Anti-Lys 8 acetylated H4 antibodies stain discrete regions in polytene chromosomes before heat shock, including the developmental puff at 62A, and nonpuffed region 89B. Heat shock loci 87A and 93D are acetylated before heat shock. $(D)$ Lys 8 acetylated $\mathrm{H} 4$ is detected at the $87 \mathrm{~A}$ and $87 \mathrm{C}$ heat shock puffs following heat shock. Chromosomal subdivision 89B, which does not contain heat shock genes, remains $\mathrm{H} 4$ acetylated during heat shock.

shock did not significantly affect the observed distribution of Lys 8 acetylated $\mathrm{H} 4$ in polytene chromosomes (Fig. 2D). Taken together, the above results suggest that the acetylation state of $\mathrm{H} 3$ and $\mathrm{H} 4$ does not change substantially during heat shock and that a gene locus can be acetylated when it is not actively transcribed.

\section{Distribution of phosphorylated histone H3 changes in response to heat shock}

The absence of a drastic change in $\mathrm{H} 3$ acetylation during heat shock was rather surprising, given current models that indicate that $\mathrm{H} 3$ acetylation is a crucial step in transcription initiation (Cheung et al. 2000; Clayton et al. 2000). This would lead us to expect that the heat shock loci would not be acetylated before heat shock and should become intensely acetylated during thermal stress. To determine if other histone modifications occur during the heat shock response, we examined whether changes in histone H3 phosphorylation occur after temperature elevation. Stimulation of quiescent cells with EGF leads to rapid and transient phosphorylation of histone $\mathrm{H} 3$ at Ser 10 of the $\mathrm{N}$-terminal arm in vivo (Thomson et al. 1999). This EGF-mediated phosphorylation of H3 is targeted to a small subpopulation of total histone H3 that is acetylated at the Lys 14 position (Clayton et al. 2000). In addition, in vitro studies have shown that phosphorylated H3 may serve as an affinity-increasing substrate for HAT activity in H3 acetylation, which raises the possibility that phosphorylation may be tied to transcription (Cheung et al. 2000). If histone phosphorylation were implicated in transcription, then the distribution of phosphorylated $\mathrm{H} 3$ might change in response to heat shock and would most likely be localized primarily to the heat shock puffs while disappearing from other loci after heat shock. Because histone H3 phosphorylation is a robust marker for mitotic cells (Hendzel et al. 1997), analysis of the distribution of phosphory- 
lated H3 in polytene chromosomes, rather than isolation of phosphorylated $\mathrm{H} 3$ from whole cell extracts, gives us the advantage of examining phosphorylation of $\mathrm{H} 3$ in a nonmitotic environment (Rudkin 1972). To examine whether the heat shock-induced puffs contain N-terminal phosphorylated H3 molecules, polytene chromosomes were stained with antibodies specific for Ser 10 phosphorylated histone H3 (Hendzel et al. 1997). Before heat shock, phosphorylated H3 staining was found in discrete bands throughout the chromosomes, with the most intense staining observed in the naturally occurring ecdysone-induced developmental puffs (Fig. 2A,G; Ashburner 1972; Bonner and Pardue 1977). After a 20min heat shock at $37^{\circ} \mathrm{C}$, phosphorylated $\mathrm{H} 3$ staining was not distributed throughout the chromosomes but was instead concentrated at a few specific sites (Fig. 2B,H). The most prominent of these regions corresponded to chromosomal divisions 63BC, 67B, and 87AC. These regions contain the hsp83 gene, the hsp22, hsp23, hsp26, and hsp27 gene cluster, and hsp70 gene clusters, respectively (Livak et al. 1978; Holmgren et al. 1981). These regions become reproducibly puffed during the heat shock response (Ashburner and Bonner 1979). Although in some chromosomes examined there were several nonheat shock loci that remained slightly phosphorylated during heat shock, the intensity of staining at these regions was much lower than the staining observed at the heat shock loci (Fig. 2B,H). The regions of the chromosome where the heat shock genes are located do not contain histone $\mathrm{H} 3$ phosphorylated at Ser 10 before heat shock (Fig. 2C,F,I). After temperature elevation, the only puffs that possessed phosphorylated histone $\mathrm{H} 3$ were the heat shock puffs. The appearance of phosphorylated histone $\mathrm{H} 3$ in the heat shock puffs, accompanied by the nearly complete reduction of staining at all other loci during heat shock, leads us to conclude that the presence of the Ser 10 phosphorylated isoform of histone H3 might be required for the transcriptional activation of the heat shock genes.

\section{Distribution of phosphorylated H3 changes dynamically during heat shock}

Induction of the heat shock genes and cessation of normal gene expression is rapid and reproducible in response to heat shock (Ashburner and Bonner 1979). Transcription run-on assays reveal that after only $1 \mathrm{~min}$ at $37^{\circ} \mathrm{C}$, the levels of many normal cellular gene transcripts have greatly diminished, with the heat shock gene transcripts dominating the population of total mRNA in the cell (Spradling et al. 1975; Ashburner and Bonner 1979). Following a heat shock, the normal pattern of gene expression within the cell is restored gradually over time (Lindquist 1986). We therefore examined the change in phosphorylated histone $\mathrm{H} 3$ staining over time during and after heat shock, to determine whether or not appearance of phosphorylated H3 closely parallels the induction of transcription of the heat shock genes and whether or not the non-heat shocked H3 distribution might be restored following recovery from heat shock (Fig. 3). After only $1 \mathrm{~min}$ at $37^{\circ} \mathrm{C}$, there is a noticeable change in the distribution of Ser 10 phosphorylated H3. The level of global H3 phosphorylation decreased, with several regions remaining intensely phosphorylated (Fig. 4B). Within $5 \mathrm{~min}$ of incubation at $37^{\circ} \mathrm{C}$, many of the less intense regions of staining have disappeared (Fig. 4C). By $10 \mathrm{~min}$ at $37^{\circ} \mathrm{C}$, the only remaining intense regions of staining are those at the heat shock puffs (Fig. 4D). When larvae were allowed to recover at room temperature from a $20-\mathrm{min}$ heat shock at $37^{\circ} \mathrm{C}, \mathrm{H} 3$ phosphorylation reappeared in several non-heat shock loci after $10 \mathrm{~min}$ of recovery (Fig. $4 \mathrm{H}$ ). After $30 \mathrm{~min}$ of recovery from heat shock, the number and distribution of loci that contained phosphorylated $\mathrm{H} 3$ appeared to be virtually indistinguishable from normal (i.e., non-heat shocked) chromosomes (Fig. 4I). This restoration of the normal (non-heat shocked) H3 phosphorylation pattern closely mimics previously described restoration of normal gene expression in cells experiencing thermal stress (Lindquist 1986).

\section{Histone H3 phosphorylation requires functional transcription factor activity}

During heat shock, the heat shock transcription factor (HSF) rapidly trimerizes in solution, localizes to the heat shock loci, binds to heat shock response promoter elements (HSEs), and induces the expression of the heat shock gene products (Westwood and Wu 1993; Jedlicka et al. 1997). The appearance of phosphorylated H3 at the heat shock loci could therefore be due to HSF recruitment of a specific histone kinase on binding to the HSEs of the heat shock genes. To test this hypothesis, we examined the staining pattern of phosphorylated H3 in polytene chromosomes isolated from $h s f^{4}$-mutant larvae, which lack functional HSF at restrictive temperatures and do not respond to thermal stress (Jedlicka et al. 1997). Before heat shock, the distribution of phosphorylated $\mathrm{H} 3$ in $h s f^{4}$-mutant chromosomes was similar to wild-type chromosomes, with staining observed in discrete bands and at the developmental puffs (Fig. 5A). In contrast to wild-type chromosomes, histone $\mathrm{H} 3$ at the heat shock loci did not become phosphorylated in $h s f^{4}$ mutant chromosomes during heat shock, which suggests that phosphorylation of histone $\mathrm{H} 3$ at the heat shock loci depends on functional HSF activity (Fig. 5B). In addition, no $\mathrm{H} 3$ phosphorylation was detected in the rest of the genome during heat shock in $h s f^{4}$ mutants, suggesting that repression of normal transcription and loss of $\mathrm{H} 3$ phosphorylation at non-heat shock loci does not require the presence of an active HSF protein.

To determine if the loss of the HSF transcription factor could also alter the distribution of acetylated $\mathrm{H} 3$ and $\mathrm{H} 4$ during heat shock, we examined acetylation of each of these histones in $h s f^{4}$-mutant polytene chromosomes. The distribution of Lys 14 acetylated histone H3 before and after heat shock in $h s f^{4}$ mutants was indistinguishable from the wild-type distribution, with staining observed at both the developmental puffs and nonpuffed regions (Fig. 5C,D). H3 acetylation was observed at the 

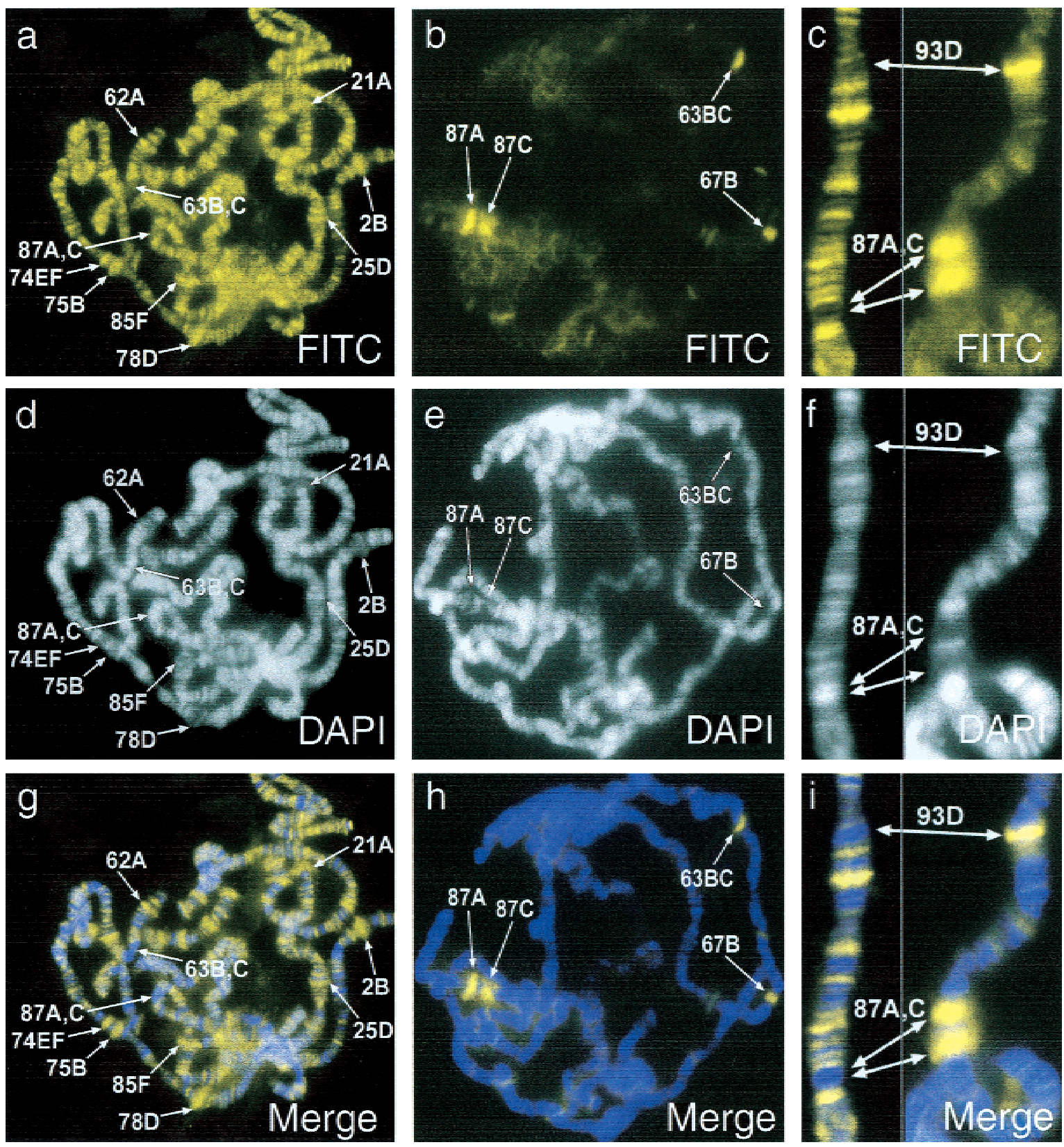

Figure 3. Distribution of phosphorylated H3 changes during heat shock. $(A-C)$ FITC image of anti-Ser 10 phosphorylated H3 immunostaining. $(D-F)$ 4',6-diamidino-2-phenylindole (DAPI) counterstaining of chromosomes shown in $(A-C)$. $(G-I)$ Composite merge of FITC and DAPI channels, antibody staining shown in yellow, DAPI shown in blue. $(A, D, G)$ Antibodies specific for Ser 10 phosphorylated $\mathrm{H} 3$ stain multiple discrete regions of polytene chromosomes isolated from third instar larvae at $22^{\circ} \mathrm{C}$, including several of the indicated ecdysone-induced developmental puffs. $(B, E, H)$ After heat shock, phosphorylated $\mathrm{H} 3$ staining is restricted to subdivisions 87AC, 63BC, and 67B, which contain the hsp70 gene cluster, hsp83 gene, and the small hsp gene cluster (hsp22, hsp23, hsp26, and hsp27), respectively. $(C, F, I)$ Image of a section of chromosome $3 \mathrm{R}$ showing the change in staining of three of the heat shock loci before and after heat shock. Heat shocked chromosome is on the right.

87A and 87C chromosomal subdivisions, which normally are puffed during heat shock but these regions do not become puffed in $h s f^{4}$-mutant chromosomes (Lis et al. 2000). Examination of acetylated $\mathrm{H} 3$ using antibodies for Lys 9- and Lys 14-acetylated H3 shows a pattern similar to that observed for the Lys 14 acetylated H3 antibody (Fig. 5E,F). In addition, $\mathrm{H} 4$ acetylation did not change after heat shock in $h s f^{4}$ mutants (Fig. 5G,H). Because the heat shock genes are not induced in $h s f^{4}$ mutants during thermal stress and because $h s f^{4}$-mutant chromosomes are acetylated, but not phosphorylated after heat shock, we conclude that $\mathrm{H} 3$ phosphorylation, and not acetylation, depends on the presence of a functional heat shock transcription factor. 

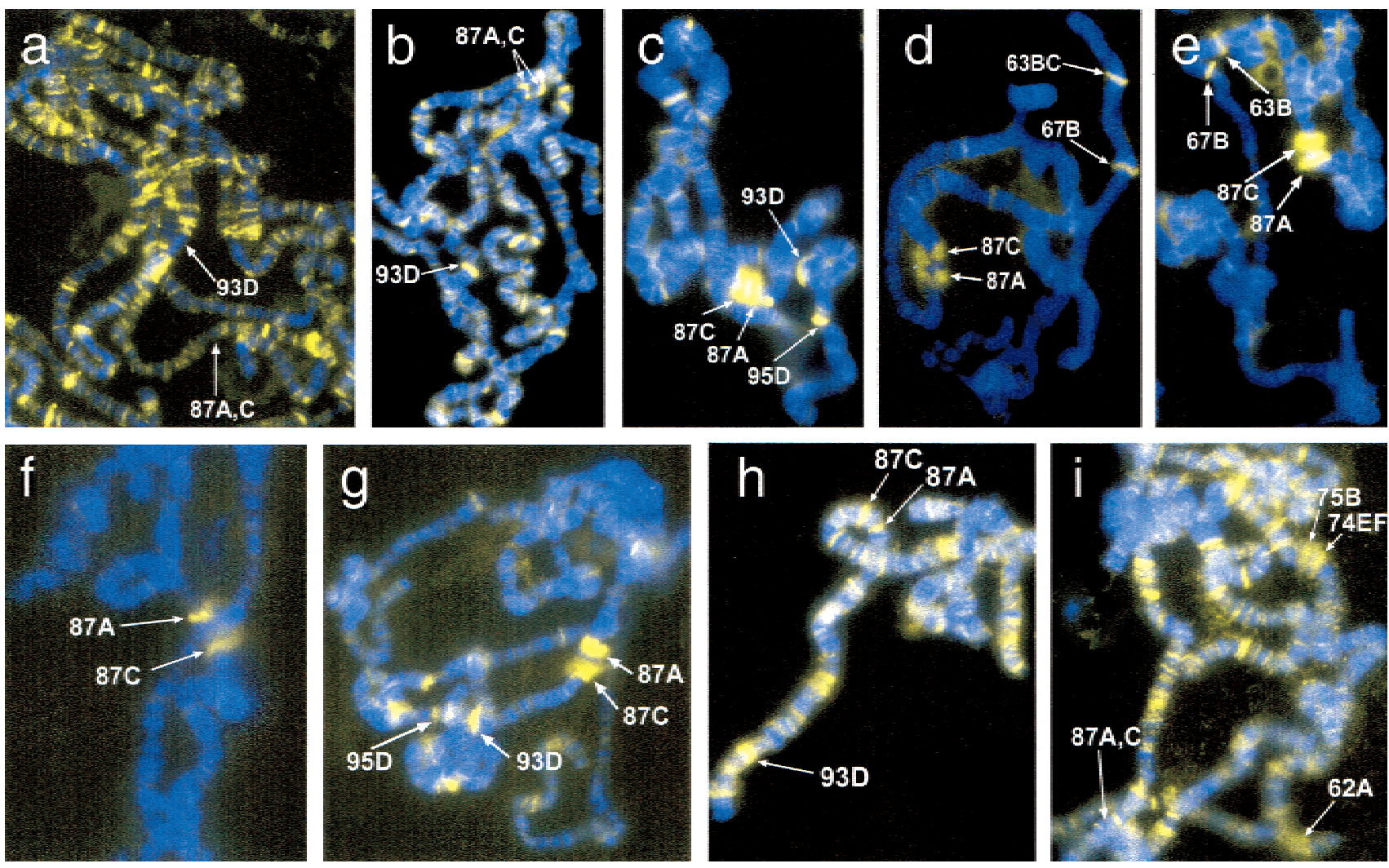

Figure 4. Distribution of antiphosphorylated histone $\mathrm{H} 3$ staining changes dynamically during and after recovery from heat shock. Visible major heat shock loci are indicated. In all panels, antibody staining is shown in yellow, chromosomes are counterstained with 4',6-diamidino-2-phenylindole (DAPI; blue). Polytene spreads were prepared from Oregon R larvae incubated for $(A) 0$ min, $(B) 1 \mathrm{~min}$, $(C) 5 \mathrm{~min},(D) 10 \mathrm{~min}$, and $(E) 20 \mathrm{~min}$ at $37^{\circ} \mathrm{C}$. Larvae were removed from $37^{\circ} \mathrm{C}$ and allowed to recover for $(F) 5 \mathrm{~min},(G) 10 \mathrm{~min},(H)$ $20 \mathrm{~min}$, and $(I) 30 \mathrm{~min}$ at room temperature.

\section{Discussion}

The results presented here suggest that phosphorylation, rather than acetylation, of the $\mathrm{N}$-terminal arm of histone H3 may correlate with an actively transcribing locus. This conclusion is based on the following observations. First, regions of heavy $\mathrm{H} 3$ phosphorylation were observed in vivo to correspond with the developmental and heat shock-induced puffs, which are described sites of active gene transcription (Ashburner 1972; Spradling et al. 1975; Ashburner and Bonner 1979). Second, the distribution and amount of phosphorylated $\mathrm{H} 3$ changed dynamically with respect to a known transcriptional stimulus (e.g., heat shock). During this same process, acetylation of residues of $\mathrm{H} 3$ and $\mathrm{H} 4$, which are important for transcription (Cheung et al. 2000; Strahl and Allis 2000), remained static. Finally, studies using HSF confirm that $\mathrm{H} 3$ phosphorylation, unlike acetylation, is dependent on the activity of a functional heat shock transcription factor. Although regions on polytene chromosomes that displayed the strongest anti-Ser 10 phosphorylated H3 staining were puffed regions, it remains to be seen whether other nonpuffed loci that were weakly stained by this antibody also contain actively transcribing genes. It must be noted that the $\mathrm{N}$-terminal arm of histone $\mathrm{H} 3$ is phosphorylated at two residues, Ser 10 and
Ser 28, both of which have been identified as having roles in mitotic chromosomal condensation (Strahl and Allis 2000). It is entirely possible that Ser 28 of histone H3 is also phosphorylated during transcription, because the antibody used in these studies is specific for Ser 10 phosphorylation (Hendzel et al. 1997) and might not have detected this particular modification.

Although previous studies performed in vitro suggest that the acetylation of histone $\mathrm{H} 3$ at a particular locus may require prephosphorylated $\mathrm{H} 3$ at that locus (Cheung et al. 2000; Lo et al. 2000), earlier studies have shown that mitogen-stimulated $\mathrm{H} 3$ phosphorylation is targeted to a small, hyperacetylated fraction of $\mathrm{H} 3$ (Barratt et al. 1994). The data presented here tend to support the latter finding in that loci that were $\mathrm{H} 3$ phosphorylated after heat shock were also H3- and $\mathrm{H} 4$-acetylated before heat shock. In addition, these data support prior conclusions (Cheung et al. 2000; Clayton et al. 2000) about the requirement of the dimodified (i.e., acetylated and phosphorylated) isoform of histone $\mathrm{H} 3$ during transcription because the heat shock puffs in wild-type polytene chromosomes also contain acetylated $\mathrm{H} 3$ and $\mathrm{H} 4$ after heat shock. However, our data suggest that the acetylation of $\mathrm{H} 3$ and $\mathrm{H} 4$ does not require HSF activity as their acetylation state does not change substantially during heat shock. It is also possible that the genes expressed during 

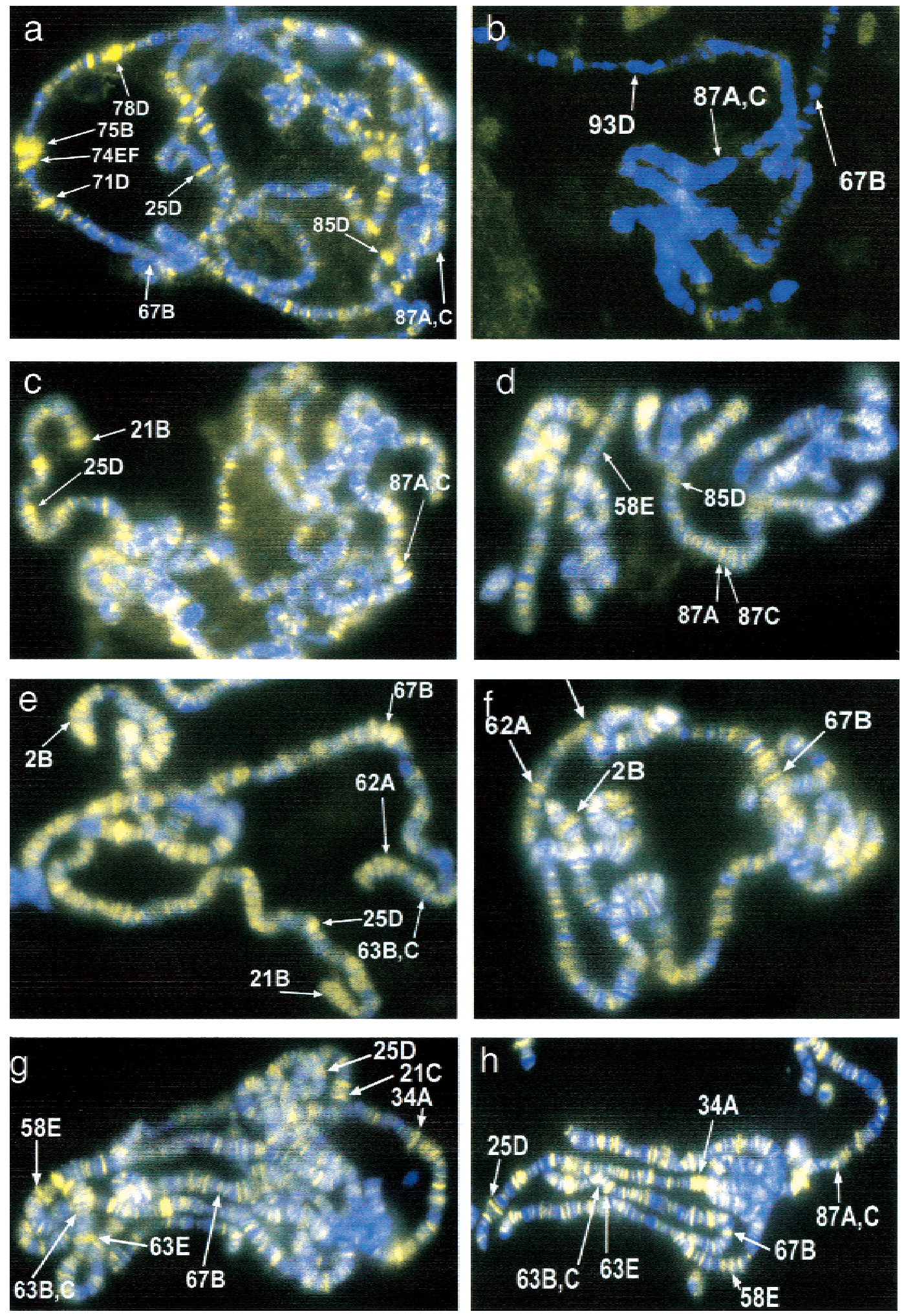

Figure 5. Analysis of $\mathrm{H} 3$ and $\mathrm{H} 4$ acetylation and $\mathrm{H} 3$ phosphorylation in hsf $f^{4}$ mutants during heat shock. In all panels, antibody staining is shown in yellow, chromosomes are counterstained with 4',6-diamidino-2-phenylindole (DAPI; blue). (A) H3 phosphorylation before heat shock appears identical to wild-type distribution. $(B)$ After heat shock, no H3 phosphorylation can be detected. $(C)$ The distribution of Lys 14 acetylated histone $\mathrm{H} 3$ does not appear to change before or $(D)$ after heat shock. $(E)$ The distribution of diacetylated H3 in $h s f^{4}$ mutant chromosomes before and $(F)$ after heat shock does not appear to change during heat shock. $(G)$ In addition, histone $\mathrm{H} 4$ acetylated at lysine 8 does not change in $h s f^{4}$ mutants before and $(H)$ after heat shock. 
A

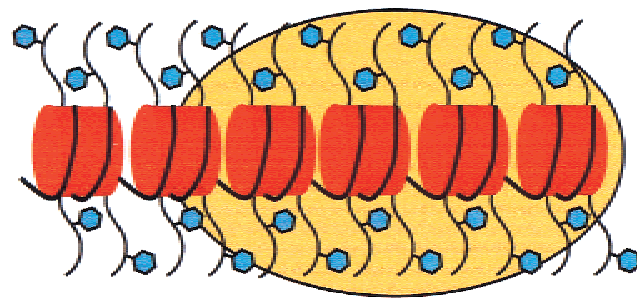

B

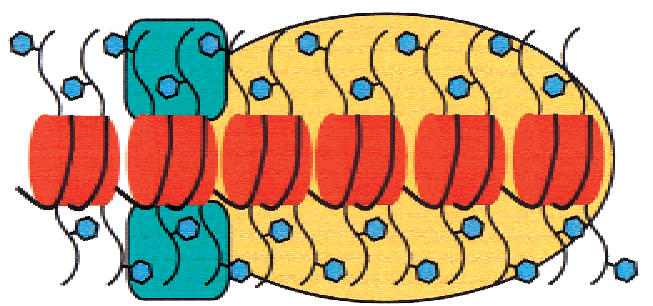

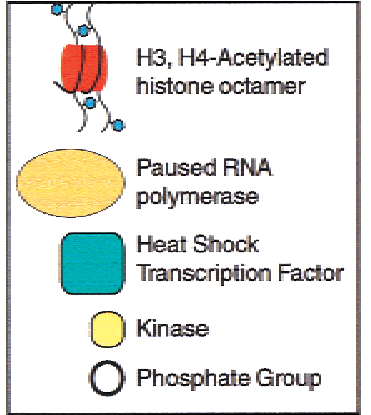

C

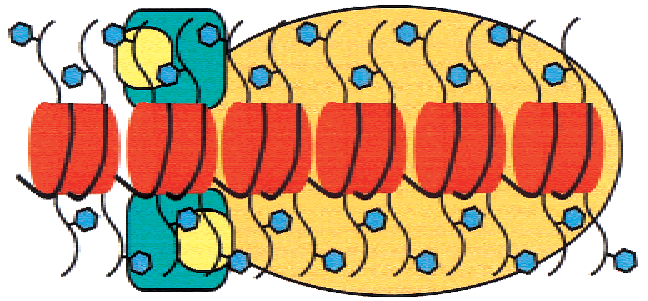

D

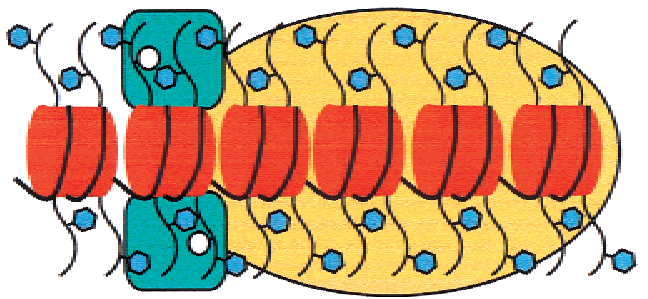

Figure 6. Model of $\mathrm{H} 3$ phosphorylation and transcription initiation during heat shock. $(A)$ The paused RNA polymerase complex present at a representative heat shock locus. $(B)$ During heat shock, the heat shock transcription factor (HSF) binds to acetylated H3 and $\mathrm{H} 4$ residues at the heat shock promoter elements. $(C)$ Bound HSF recruits a histone kinase, which $(D)$ phosphorylates histone H3 at the Ser 10 position.

the heat shock response represent a special case that may require a more rapid means of induction of expression in contrast to the relatively slow process of phosphorylation followed by acetylation and deacetylation /Clayton et al. 2000). Further studies need to be performed to determine whether these $\mathrm{H} 3$ modifications are restricted to histones within the promoter region or histones found throughout the entire gene.

How might acetylation and phosphorylation of histones $\mathrm{H} 3$ and $\mathrm{H} 4$ work together to promote transcription of a particular gene? Our data suggest that acetylated histones might define a particular locus that is primed for possible phosphorylation and subsequent transcription (Fig. 6A). This acetylated locus would attract transcription factors (Fig. 6B) that interact with the acetylated residues on histones $\mathrm{H} 3$ and $\mathrm{H} 4$, known to be essential for proper association of several transcription factors with their promoters (Vettese-Dadey et al. 1996; Howe et al. 1999; Jacobson et al. 2000). Once bound to this locus, the transcription factor would then recruit a particular histone kinase (Fig. 6C), which phosphorylates Ser 10 of the N-terminal arm of histone H3 (Fig. 6D). The most logical site of phosphorylation would be an $\mathrm{H} 3$ molecule with a Lys 14 acetylated N-terminal arm, a species that has been shown to exist in vivo /Clayton et al. 2000). The presence of this dimodified H3 would define that locus as "active" for transcription.

There are several kinases known to localize to specific loci on polytene chromosomes that phosphorylate $\mathrm{H} 3$ in vitro, such as JIL-1 on the X chromosome (Jin et al. 1999) and P-TEFb kinase at the heat shock loci (Lis et al. 2000). This raises the possibility that the specificity of a kinase for activation of a particular gene through H3 phosphorylation might be regulated by the specific transcription factors that control expression of this gene. We have yet to determine whether phosphorylation of $\mathrm{H} 3$ is required for assembly of the RNA polymerase II complex or if phosphorylation is a by-product of complex formation and polymerase procession during transcription. If phosphorylation of $\mathrm{H} 3$ were indeed the critical step in activating gene transcription, then a reasonable hypothesis is that deactivation of a particular gene would be dependent on either regulated or unregulated phosphatase activity to remove the activating phosphate group from the $\mathrm{N}$-terminal tails of H3. The disappearance of phosphorylated $\mathrm{H} 3$ at nontranscribing loci and appearance of phosphorylated $\mathrm{H} 3$ at actively transcribing loci during heat shock suggests that a functional transcription complex might actively maintain the phosphorylated state of histone $\mathrm{H} 3$, which would be subject to ready dephosphorylation by either passive or regulated phosphatase activity in a nontranscribing state.

\section{Materials and methods}

Stocks were maintained in standard medium at $22^{\circ} \mathrm{C}$. Oregon $\mathrm{R}$ larvae were used for the wild-type controls in all experiments. For induction of the heat shock response, wandering third instar larvae were placed in $1.5-\mathrm{mL}$ microcentrifuge tubes with punctured caps. The microcentrifuge tubes were then incubated for $20 \mathrm{~min}$ in a water bath maintained at $37^{\circ} \mathrm{C}$. After removal from the water bath, salivary glands were immediately isolated under PBS containing $4 \%$ paraformaldehyde to prevent temperature recovery. Salivary glands isolated from wandering third instar Oregon $\mathrm{R}$ and $\mathrm{cn} \mathrm{br}$; $h s f^{4}$ larvae were fixed in $4 \%$ paraformaldehyde, squashed in $45 \%$ acetic acid on subbed slides (Ashburner 1989). The slides were frozen in liquid nitrogen, and stored dry at $-70^{\circ} \mathrm{C}$ until used.

Slides were incubated overnight in antibody dilution buffer 
$(1 \times$ PBS; $1 \%$ BSA; $0.05 \%$ Triton $\mathrm{X}-100)$ containing primary antibodies at $1: 100$ (antiphosphohistone H3) or 1:25 (antiacetylated histone H3, Lys 14; antiacetylated histone H3, Lys 9 and Lys 14; and antiacetylated histone H4, Lys 8). All primary antibodies used in this study were purchased from Upstate Biotechnologies. Following primary incubation, slides were washed three times in antibody dilution buffer, and incubated with a $1: 250$ dilution of FITC conjugated goat antirabbit IgG (Jackson Immunoresearch Laboratories) for $1 \mathrm{~h}$ at $37^{\circ} \mathrm{C}$. Slides were washed three times in antibody dilution buffer, rinsed briefly in PBS, stained with 4',6-diamidino-2-phenylindole (DAPI), $0.5 \mu \mathrm{g} / \mathrm{mL}$, and mounted in Vectashield antifade mounting medium (Vector Laboratories) for viewing.

\section{Acknowledgments}

We thank Dr. Tatiana Gerasimova for advice with cytological analysis, Xiaoyuan Lu for initial work on the project, and Drs. Mariano Labrador and Fabien Mongelard for frank discussion and critical comments on the manuscript. $c n b r$; $h s f^{4}$ flies were kindly provided by Dr. Carl Wu. This work was supported by Public Health Service grant GM35463 from the National Institutes of Health.

The publication costs of this article were defrayed in part by payment of page charges. This article must therefore be hereby marked "advertisement" in accordance with 18 USC section 1734 solely to indicate this fact.

\section{References}

Ashburner, M. 1970. Patterns of puffing activity in the salivary gland chromosomes of Drosophila. V. Responses to environmental treatments. Chromosoma 31: 356-376.

1972. Puffing patterns in Drosophila melanogaster and related species. Results Probl. Cell Differ. 4: 101-151.

- 1989. Drosophila. Cold Spring Harbor Laboratory Press, Cold Spring Harbor, NY.

Ashburner, M. and Bonner, J.J. 1979. The induction of gene activity in Drosophila by heat shock. Cell 17: 241-254.

Barratt, M.J., Hazzalin, C.A., Cano, E., and Mahadevan, L.C. 1994. Mitogen-stimulated phosphorylation of histone H3 is targeted to a small hyperacetylation-sensitive fraction. Proc. Nat1. Acad. Sci. 91: 4781-4785.

Berger, S.L. 1999. Gene activation by histone and factor acetyltransferases. Curr. Opin. Cell. Biol. 11: 336-341.

Boggs, B.A., Connors, B., Sobel, R.E., Chinault, A.C., and Allis, C.D. 1996. Reduced levels of histone H3 acetylation on the inactive $\mathrm{X}$ chromosome in human females. Chromosoma 105: 303-309.

Bonner, J.J. and Pardue, M.L. 1977. Ecdysone-stimulated RNA synthesis in salivary glands of Drosophila melanogaster: Assay by in situ hybridization. Cell 12: 219-225.

Cheung, P., Tanner, K.G., Cheung, W.L., Sassone-Corsi, P., Denu, J.M., and Allis, C.D. 2000. Synergistic coupling of histone $\mathrm{H} 3$ phosphorylation and acetylation in response to epidermal growth factor stimulation. Mol. Cell. 5: 905-915.

Clayton, A.L., Rose, S., Barratt, M.J., and Mahadevan, L.C. 2000. Phosphoacetylation of histone $\mathrm{H} 3$ on c-fos- and c-jun-associated nucleosomes upon gene activation. EMBO $J$. 19: 3714-3726.

Hendzel, M.J., Wei, Y., Mancini, M.A., Van Hooser, A., Ranalli, T., Brinkley, B.R., Bazett-Jones, D.P., and Allis, C.D. 1997. Mitosis-specific phosphorylation of histone $\mathrm{H} 3$ initiates primarily within pericentromeric heterochromatin during G2 and spreads in an ordered fashion coincident with mitotic chromosome condensation. Chromosoma 106: 348-360.

Holmgren, R., Corces, V., Morimoto, R., Blackman, R., and Meselson, M. 1981. Sequence homologies in the $5^{\prime}$ regions of four Drosophila heat-shock genes. Proc. Natl. Acad. Sci. 78: 3775-3778.

Howe, L., Brown, C.E., Lechner, T., and Workman, J.L. 1999. Histone acetyltransferase complexes and their link to transcription. Crit. Rev. Eukaryot. Gene Expr. 9: 231-243.

Jacobson, R.H., Ladurner, A.G., King, D.S., and Tjian, R. 2000. Structure and function of a human TAF(II)250 double bromodomain module. Science 288: 1422-1425.

Jedlicka, P., Mortin, M.A., and Wu, C. 1997. Multiple functions of Drosophila heat shock transcription factor in vivo. EMBO J. 16: 2452-2462.

Jin, Y., Wang, Y., Walker, D.L., Dong, H., Conley, C., Johansen, J., and Johansen, K.M. 1999. JIL-1: A novel chromosomal tandem kinase implicated in transcriptional regulation in Drosophila. Mol. Cell 4: 129-135.

Kuo, M.H., Brownell, J.E., Sobel, R.E., Ranalli, T.A., Cook, R.G., Edmondson, D.G., Roth, S.Y., and Allis, C.D. 1996. Transcription-linked acetylation by Gen5p of histones H3 and H4 at specific lysines. Nature 383: 269-272.

Lindquist, S. 1986. The heat-shock response. Annu. Rev. Biochem. 55: 1151-1191.

Lis, J.T., Mason, P., Peng, J., Price, D.H., and Werner, J. 2000. $\mathrm{P}-\mathrm{TEFb}$ kinase recruitment and function at heat shock loci. Genes \& Dev. 14: 792-803.

Livak, K.J., Freund, R., Schweber, M., Wensink, P.C., and Meselson, M. 1978. Sequence organization and transcription at two heat shock loci in Drosophila. Proc. Natl. Acad. Sci. 75: 5613-5617.

Lo, W.-S., Trievel, R.C., Rojas, J.R., Duggan, L., Hsu, J.-Y., Allis, C.D., Marmorstein, R., and Berger S.L. 2000. Phosphorylation of serine 10 in histone $\mathrm{H} 3$ is functionally linked in vitro to Gcn5-mediated acetylation at lysine 14. Mol. Cell 5: 917926.

Mahadevan, L.C., Willis, A.C., and Barratt, M.J. 1991. Rapid histone $\mathrm{H} 3$ phosphorylation in response to growth factors, phorbol esters, okadaic acid, and protein synthesis inhibitors. Cell 65: 775-783.

McKenzie, S.L., Henikoff, S., and Meselson, M. 1975. Localization of RNA from heat-induced polysomes at puff sites in Drosophila melanogaster. Proc. Natl. Acad. Sci. 72: 11171121.

Mirault, M.E., Goldschmidt-Clermont, M., Moran, L., Arrigo, A.P., and Tissieres, A. 1978. The effect of heat shock on gene expression in Drosophila melanogaster. Cold Spring Harb. Symp. Quant. Biol. 42: 819-827.

Mizzen, C.A. and Allis, C.D. 1998. Linking histone acetylation to transcriptional regulation. Cell. Mol. Life Sci. 54: 6-20.

Mizzen, C.A., Yang, X.J., Kokubo, T., Brownell, J.E., Bannister, A.J., Owen-Hughes, T., Workman, J., Wang, L., Berger, S.L., Kouzarides, T., et al. 1996. The TAF(II)250 subunit of TFIID has histone acetyltransferase activity. Cell 87: 1261-1270.

Nan, X., Ng, H.H., Johnson, C.A., Laherty, C.D., Turner, B.M., Eisenman, R.N., and Bird, A. 1998. Transcriptional repression by the methyl-CpG-binding protein MeCP2 involves a histone deacetylase complex. Nature 393: 386-389.

Nightingale, K.P., Wellinger, R.E., Sogo, J.M., and Becker, P.B. 1998. Histone acetylation facilitates RNA polymerase II transcription of the Drosophila hsp26 gene in chromatin. EMBO T. 17: 2865-2876.

Pauli, D., Arrigo, A.P., and Tissieres, A. 1992. Heat shock response in Drosophila. Experientia 48: 623-629.

Rudkin, G.T. 1972. Replication in polytene chromosomes. Re- 
sults Probl. Cell. Differ. 4: 59-85.

Smith, E.R., Pannuti, A., Gu, W., Steurnagel, A., Cook, R.G., Allis, C.D., and Lucchesi, J.C. 2000. The Drosophila MSL complex acetylates histone $\mathrm{H} 4$ at lysine 16, a chromatin modification linked to dosage compensation. Mol. Cell. Biol. 20: 312-318.

Spradling, A., Penman, S., and Pardue, M.L. 1975. Analysis of Drosophila mRNA by in situ hybridization: Sequences transcribed in normal and heat shocked cultured cells. Cell 4: 395-404.

Strahl, B.D. and Allis, C.D. 2000. The language of covalent histone modifications. Nature 403: 41-45.

Thomson, S., Clayton, A.L., Hazzalin, C.A., Rose, S., Barratt, M.J., and Mahadevan, L.C. 1999. The nucleosomal response associated with immediate-early gene induction is mediated via alternative MAP kinase cascades: MSK1 as a potential histone H3/HMG-14 kinase. EMBO J. 18: 4779-4793.

Trievel, R.C., Rojas, J.R., Sterner, D.E., Venkataramani, R.N., Wang, L., Zhou, J., Allis, C.D., Berger, S.L., and Marmorstein, R. 1999. Crystal structure and mechanism of histone acetylation of the yeast GCN5 transcriptional coactivator. Proc. Natl. Acad. Sci. 96: 8931-8936.

Turner, B.M. 1991. Histone acetylation and control of gene expression. J. Cell. Sci. 99: 13-20.

Vettese-Dadey, M., Grant, P.A., Hebbes, T.R., Crane- Robinson, C., Allis, C.D., and Workman, J.L. 1996. Acetylation of histone $\mathrm{H} 4$ plays a primary role in enhancing transcription factor binding to nucleosomal DNA in vitro. EMBO J. 15: 2508 2518.

Wei, Y., Mizzen, C.A., Cook, R.G., Gorovsky, M.A., and Allis, C.D. 1998. Phosphorylation of histone H3 at serine 10 is correlated with chromosome condensation during mitosis and meiosis in Tetrahymena. Proc. Nat1. Acad. Sci. 95: 7480-7484.

Wei, Y., Yu, L., Bowen, J., Gorovsky, M.A., and Allis, C.D. 1999. Phosphorylation of histone $\mathrm{H} 3$ is required for proper chromosome condensation and segregation. Cell 97: 99-109.

Westwood, J.T. and Wu, C. 1993. Activation of Drosophila heat shock factor: Conformational change associated with a monomer-to-trimer transition. Mol. Cell. Biol. 13: 34813486.

Wittschieben, B.O., Otero, G., de Bizemont, T., Fellows, J., Erdjument-Bromage, H., Ohba, R., Li, Y., Allis, C.D., Tempst, P., and Svejstrup, J.Q. 1999. A novel histone acetyltransferase is an integral subunit of elongating RNA polymerase II holoenzyme. Mol. Cell. 4: 123-128.

Wolffe, A. 1998. Chromatin: Structure and function. Academic Press, San Diego, CA. 


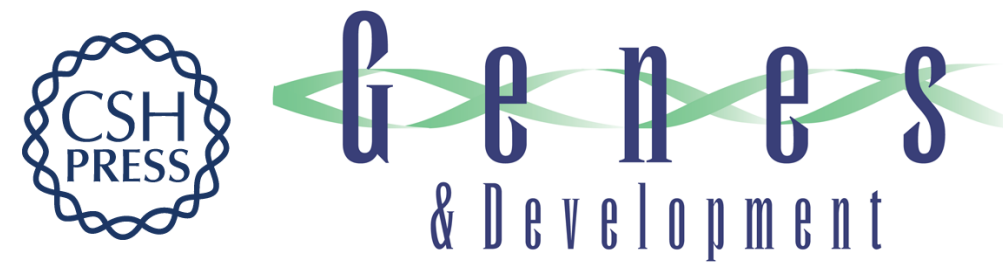

\section{Phosphorylation of histone $\mathrm{H} 3$ correlates with transcriptionally active Ioci}

Scott J. Nowak and Victor G. Corces

Genes Dev. 2000, 14:

Access the most recent version at doi:10.1101/gad.848800

References This article cites 39 articles, 15 of which can be accessed free at: http://genesdev.cshlp.org/content/14/23/3003.full.html\#ref-list-1

License

Email Alerting Receive free email alerts when new articles cite this article - sign up in the box at the top Service right corner of the article or click here.

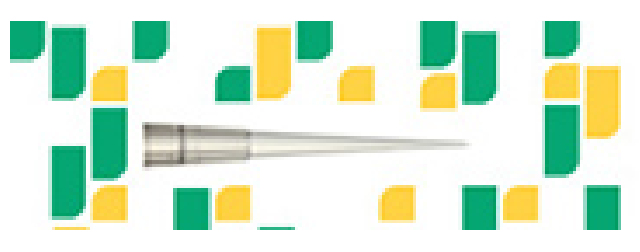

Focused on your science. 